\title{
Globalisation and Technological Convergence in the EU*
}

\author{
Camilla Mastromarco \\ Laura Serlenga \\ University of Salento, CESIfo and RCEA \\ University of Bari and IZA \\ Yongcheol Shin \\ Department of Economics and Related Studies, University of York
}

Revised, March 2012

\begin{abstract}
We employ a two-step approach in investigating the dynamic transmission channels under which globalization factors foster technical efficiency by combining a dynamic efficiency analysis in the stochastic frontier framework, and a time series approach based on VAR and spectral analysis. Using the dataset of the $18 \mathrm{EU}$ countries over 1970-2004, we find that both import and FDI are significant factors in spreading efficiency externalities and thus accelerating technology catch-up in the EU. In particular, the impacts of the import are more prominent in the short-run while those of FDI play a more important role over the longer-run. Furthermore, the impacts of the import are pro-cyclical only in the short-run whereas those of FDI are pro-cyclical mostly over the medium- to the long-run. This evidence is broadly consistent with the sample observation that the recent slowdown of the EU productivity has been closely related to the corresponding FDI decline especially after 2000 . Hence, any protection-oriented policy will be likely to be more detrimental for the EU.
\end{abstract}

JEL: D24, O47, C13, C33.

Keywords: Stochastic Frontier in Heterogeneous Panels, Time-Varying Efficiency, Globalisation Factors, Unobserved Factors, Spectral and Impulse Response Analyses

\footnotetext{
${ }^{*}$ We owe a debt of gratitude to the Editor and to two anonymous referees for their constructive feedbacks on an earlier draft of the manuscript. We are also grateful to Badi Baltagi, Charlie Cai, Kausik Chaudhuri, Ana Galvão, George Kapetanios, Junsoo Lee, Kevin Reilly, Ulrich Woitek, the session participants at the 2nd Italian Congress of Econometrics and Applied Economics at Rimini, 2007, and at the 15th Panel Data Conference at Bonn, 2009, and the seminar participants at Universities of Zurich and Leeds for their helpful comments. The third author acknowledges partial financial support from the ESRC (Grant No. RES-000-22-3161). The usual disclaimer applies.
} 


\section{Introduction}

For the first time since 1982, the world trade registered a sharp decline in 2008Q4, mainly owing to the global recession that has been triggered by the ongoing financial crisis, e.g. Greenwood-Nimmo et al. (2011). Contraction in trade also helps to spread further economic contraction initially originated from developed countries to emerging markets. Given the priority to tackle domestic economic problems, the policy makers are tempted to protect the national economy by imposing restrictions on imports, reserving government contracts for domestic firms and refusing to help companies who mainly invest abroad. For example, several protection policies have been adopted in the energy sector in France, and in the banking sector and auto industries in Germany, Italy and Spain (Economist, February 2006). However, trade is proven to be an important channel for technology transfer (Grossman and Helpman 1991, 1996 and Aghion and Howitt, 1992).

The growth literature highlights that capital accumulation and technological diffusion play an important role in promoting economic growth, e.g. Nelson and Phelps (1966), Jovanovic and Rob (1989), Romer (1990), Segerstrom (1991) and Barro and Sala-i-Martin (2004). It also attempts to uncover the transmission channels in which technology catch-ups, defined as individual countries' abilities to adopt and accumulate new technologies, will affect growth rates. Bernard and Jones (1996) demonstrate that technology catch-up will be a dominant factor in reaching the steady-state level of output growth.

In general, technological diffusion is likely to play a significant role in spurring productivity growth by lowering barriers to flows of imported goods and foreign direct investment (FDI). If knowledge transfers made available by FDI and imports create efficiency externalities, such openness is expected to raise total factor productivity through efficiency gains, e.g. Borensztein et al. (1998) and Cameron et al. (2005). Hence, efficiency improvement will represent productivity catch-up via technology diffusion because inefficiencies generally reflect a sluggish adoption of new technologies (e.g. Ahn et al., 2000). In a cross-country framework, production inefficiencies can be identified as the distance of the individual production from the frontier as proxied by the maximum output of the reference country (regarded as an empirical counterpart of an optimal production boundary).

In this paper we aim to provide a coherent measure of technical efficiency, which closely matches the concept of technology diffusion through openness channel and to assess the diffusion dynamics of a common technology (frontier) in the EU countries with respect to two openness factors: FDI and trade. We then aim to redress an important policy issue of whether the protection-oriented policy will hamper the production efficiency through limiting both FDI and import inflows by explicitly analysing dynamic interactions between common efficiency and globalisation factors in the EU. 
To this end, we employ a robust estimation procedure for dealing with dynamics of time-varying efficiency by explicitly taking into account unobserved factors in stochastic frontier panel data framework, which enables us to better capture the impacts of global shocks such as openness and trade policy on the production frontier and technical efficiency, simultaneously. Hence, our study is expected to overcome two main shortcomings associated with some studies in modelling efficiency dynamics: the complexity of the likelihood function observed in the one-stage approach by Battese and Coelli (1995) and the downward bias of the two-stage estimation documented in Wang and Schmidt (2002).

In particular, we adopt a two-step estimation approach: firstly, we extend the existing approach (e.g. Lee, 1991; Lee and Schmidt, 1992; Ahn et al., 2007; Kneip et al., 2011), and consider the panel data-based stochastic frontier model with unobserved time-varying factors in a general setting. We then adopt the pooled common correlated effects estimation technique advanced by Pesaran (2006) and extended by Serlenga and Shin (2007a), in order to obtain consistent estimates of individual and common time-varying efficiency measures. Next, we apply a trivariate VAR to modelling dynamic interactions of common efficiency and two globalization factors, proxied by FDI and imports. By applying the spectral-based dynamic correlation analysis (Croux et al., 2001; Mastromarco and Woitek, 2007) and the impulse response analysis, we can directly examine dynamic interactions between efficiency and globalization factors, through which we can get insights on the technological convergence, the business cycles of the common EU efficiency, and the technological catch-up process.

Fluctuations in efficiency may provide a reasonable indicator of the phase of business cycles since efficiency is a component of total factor productivity. Furthermore, globalisation factors can be closely related to internal and external demand and supply fluctuations (Krugman, 1991). Hence, a careful evaluation of whether factors such as trade and FDI are pro- or counter-cyclical with efficiency provides an important policy implication. To date, however, there have been no studies that attempt to directly investigate this important issue. Instead, most existing studies have partially and indirectly analysed the impacts of globalisation factors on cross-country business cycle synchronisation (e.g. Canova and Dellas, 1993; Kose et al., 2003; Artis et al., 2007). Importantly, this paper will directly address the issue of business cycle synchronisation between globalisation factors and common EU efficiency.

Using the dataset comprising the 18 EU countries over the period 1970-2004, we find the main empirical results as follows: (i) The dominant cycles of common technology efficiency of the EU countries have a length of 3 to 5 years; (ii) Imports and FDI appear to be weakly synchronised with the common efficiency cycle, though business cycles of common efficiency have a higher co-movement 
with FDI than imports at most frequencies, and especially after 5 years when the FDI impacts on efficiency are fully absorbed; (iii) The impulse response analysis shows that positive shocks to FDI and imports improve common EU efficiency, suggesting that both factors may act as an important transmission channel to diffuse common technology; (iv) The impulse responses of common EU efficiency with respect to the import and the FDI shocks reach a peak after 4 years and 9 years, respectively. Furthermore, the correlation between efficiency and FDI is higher at cycles longer than 4 years.

Our empirical findings highlight that globalization and openness factors might be considered as a driving force in spreading technical efficiency across the EU countries, and support the theoretical findings of positive externalities associated with globalization and openness as documented in Edwards (1998), Frankel and Romer (1999), Barro (2001), Barro and Sala-i-Martin (2004) and Artis et al. (2007). We also find the different time delayed effects of two factors on technology efficiency. The impacts of import shocks are significantly higher in the short run while the FDI shocks play a more important role over the medium- to the longterm in accelerating technology catch-up in the EU. Significant lagged impacts of FDI shocks confirm that the knowledge embodied in FDI requires substantially longer time period to be fully transferred for efficiency externalities, e.g. Cohen and Levinthal (1989), Tybout (1992) and Coe and Helpman (1995). Furthermore, this evidence is broadly consistent with the sample observation that both productivity and FDI have started to slow down around 2000, e.g. Van Ark et al. (2007) and Taylor (2008).

Hence, our findings support the studies highlighting that lowering barriers to entry of foreign goods and investments have exerted a significantly positive effects on productivity. We also find that imports are pro-cyclical with technical efficiency only in the short-run whereas FDI are pro-cyclical from the medium- to the longrun. Thus, an improvement in FDI and trade will be likely to enhance efficiency and promote business cycle synchronisation. Therefore, the EU countries that pursue relatively closed policies will be unlikely to gain the benefits associated with technology diffusion implicit in international movement of goods, capitals and investments, e.g. Wacziarg and Welch (2008) and ECB (2009). Hence, the protection-oriented policy will be more detrimental on technology catch-up in the EU and likely to exert further negative impacts on the global economy.

The paper is organized as follows: Section 2 overviews the literature on stochastic frontier modelling in panels and describes our proposed estimation strategies. Section 3 provides main empirical findings. Section 4 concludes. 


\section{Methodology}

An exact dynamic mechanism as to how technology efficiency and globalization factors such as FDI and trade relate to each other remains ambiguous and neglected in the literature at least on empirical grounds. In this regard, we suggest to examine the dynamic transmission mechanism under which globalization factors foster technical efficiency. To this end we combine a dynamic efficiency analysis in the stochastic frontier framework and the time series vector autoregressive (VAR) analysis. In the first step, we employ the generalised stochastic frontier specification with unobserved factors (thus incorporating the cross section dependence), and obtain consistent estimates of individual and common technical efficiency. In the second step, we model dynamic interactions between common efficiency and globalization factors through the spectral analysis and the impulse response functions. Hence, our study is expected to deal with an important research question if an open (and not protection-oriented) policy will be likely to foster technological convergence in the EU countries through a careful analysis of the dynamic effects of trade and investment liberalisation on the EU efficiency.

\subsection{Estimation of Time-varying Common EU Efficiency}

The relationship between economic growth and technology diffusion remains a conundrum. Particularly at the center of theoretical debates, lies the issue as to how best to model dynamic adjustments. However, the stochastic frontier approach (SFA), as pioneered by Aigner et al. (1977) and Meeusen and van den Broeck (1977), tends to focus on estimating the long-run equilibrium relationship between output and production factors without explicitly modelling time varying patterns of inefficiency (e.g. Kumbhakar and Lovell, 2000). In particular, the partial adjustment process cannot be appropriately encapsulated by a standard frontier model based on an implicit assumption of complete adjustment because the adjustment from the current to the desired future input use is far from perfect due to time delays, delivery lags and installation costs. This neglect may result in a misleading conclusion that an intertemporally efficient producer can be classified as being inefficient.

To capture the time-varying technical efficiency more accurately, a number of alternative approaches have been proposed, e.g. Cornwell et al. (1990), Kumbhakar (1990), Battese and Coelli (1995) and Ahn et al. (2000). In particular, in panels with small time periods, Lee (1991) and Lee and Schmidt (1992) consider the framework under which individual technical inefficiencies vary over time but common to the cross-sectional unit. Ahn et al. (2007) develop an extended frontier model with multiple components. Furthermore, Kneip et al. (2011) propose a flexible factor-based approach in which time-varying individual effects can be 
represented as a linear combination of a small number of unknown basis functions with heterogeneous coefficients. This development clearly demonstrates an important role of factors in SFA.

We follow these trends, and consider the panel stochastic frontier model:

$$
y_{i t}=\beta^{\prime} \mathbf{x}_{i t}+\delta^{\prime} \mathbf{s}_{i t}+\varepsilon_{i t}, i=1, \ldots, N, t=1, \ldots, T
$$

with the two-way error components structure given by

$$
\varepsilon_{i t}=v_{i t}-u_{i t}=v_{i t}-\left(\alpha_{i}+b_{i} t+\varphi_{i} \theta_{t}\right),
$$

where $y_{i t}$ is a logarithm of output of country $i$ at time $t, \mathbf{x}_{i t}$ a $k \times 1$ vector of logged production inputs, $\mathbf{s}_{i t}$ an $s \times 1$ vector of global variables such as FDI and imports of individual countries, ${ }^{1} \beta$ and $\delta$ are $k$ - and $s$ - vectors of parameters, and $\varepsilon_{i t}$ the composite stochastic error including the idiosyncratic disturbance $\left(v_{i t}\right)$ and the term measuring the (time-varying) technical inefficiency. We then follow Schmidt and Sickles (1984), and attempt to measure individual technical inefficiency by

$$
e_{i t}=\max _{i} u_{i t}-u_{i t}=\max _{i}\left(\alpha_{i}+b_{i} t+\varphi_{i} \theta_{t}\right)-\left(\alpha_{i}+b_{i} t+\varphi_{i} \theta_{t}\right), t=1, \ldots, T .
$$

Notice that the specification (2) can be regarded as a special case of the $p$-factor model considered by Ahn et al. (2007) with $p=3$ and three factors of $\left(1, t, \theta_{t}\right)$. It is more general than the model by Cornwell et al. (1990) with three factors of $\left(1, t, t^{2}\right)$ since we do not fully specify the time varying pattern of the changes in technical inefficiencies.

The time-varying technical inefficiency $\left(u_{i t}\right)$ now consists of three components: $\alpha_{i}$ is (unobserved) time-invariant individual effects, the time trend, $t$, is supposed to capture an exogenous technological change (e.g. Battese and Coelli, 1995; Ahn et al., 2000), and finally, the (unobserved) time-specific effects $\left(\theta_{t}\right)$ are expected to provide a good proxy for any remaining nonlinear and complex trending patterns associated with the globalisation and the business-cycle events. ${ }^{2}$ As the global economy becomes increasingly integrated, all the individual countries are likely to be exposed more to global shocks.

\footnotetext{
${ }^{1}$ In an earlier version of the paper, we have also considered an alternative specification in which the global variables enter the regression in (1) as the observed factors such as $\delta_{i}^{\prime} \overline{\mathbf{s}}_{t}$ or $\delta^{\prime} \overline{\mathbf{s}}_{t}$ where $\overline{\mathbf{s}}_{t}=N^{-1} \sum_{i=1}^{N} \mathbf{s}_{i t}$. In both cases, however, there is an identification problem such that technical inefficiency $\left(e_{i t}\right)$ in $(3)$ cannot be uniquely estimated. We are grateful to an anonymous referee for pointing this issue.

${ }^{2}$ Notice under the current setup that an exogenous technological change, usually assumed to be common to every individual and specified as $\gamma t$ for example, cannot be uniquely identified from $b_{i} t$ in (2), where $b_{i}$ measures an individual diffusion response to common (exogenous) technological change. We are grateful to an anonymous referee for pointing this issue. Furthermore, the use of a linear time trend cannot track the non-monotonic productivity decline as we observed from the mid 90 s in the EU, see Section 3 below.
} 
It is easily seen that most econometric specifications of the production frontier in the literature can be expressed as a variation of the model given by (1) and (2). Another distinguishing feature is that our model can also accommodate a certain degree of cross section dependence through the heterogeneous factor loadings, $\varphi_{i}$ for $i=1, \ldots, N{ }^{3}$ Hence, this factor-based approach is expected to capture the timevarying pattern of technical inefficiency in a more robust manner, especially by including $s_{i t}$ as regressors explicitly in the stochastic frontier, (1), such that it can overcome the shortcomings associated with the two step approach as considered by Wang and Schmidt (2002).

The conventional panel data estimator such as the fixed or the random effects estimators would be seriously biased without properly accommodating the error component structure given by (2), as confirmed by Monte Carlo studies by Kapetanios and Pesaran (2007). Hence, in order to consistently estimate the structural parameters, $\beta$ and $\delta$ in (1), and then technical inefficiency $\left(e_{i t}\right)$ in $(3)$, we follow the Pooled Common Correlated Effects (hereafter, PCCE) estimator advanced by Pesaran (2006), ${ }^{4}$ who shows that unobserved common factor, $\theta_{t}$, can be consistently proxied by cross-sectional averages of dependent and independent variables as $N, T \rightarrow \infty$, and $T / N \rightarrow K$ with $0 \leq K<\infty$. Taking the crosssectional average of $(1)$,

$$
\bar{y}_{t}=\beta^{\prime} \overline{\mathbf{x}}_{t}+\delta^{\prime} \overline{\mathbf{s}}_{t}+\bar{\varepsilon}_{t}=\beta^{\prime} \overline{\mathbf{x}}_{t}+\delta \overline{\mathbf{s}}_{t}+\bar{v}_{t}-\left(\bar{\alpha}+\bar{b} t+\bar{\varphi} \theta_{t}\right),
$$

it is easily seen that

$$
\theta_{t}=\frac{-1}{\bar{\varphi}}\left(\bar{y}_{t}-\beta^{\prime} \overline{\mathbf{x}}_{t}-\delta^{\prime} \overline{\mathbf{s}}_{t}+\bar{\alpha}+\bar{b} t-\bar{v}_{t}\right),
$$

where the bar over the variable denotes the cross-sectional average (e.g. $\bar{y}_{t}=$ $\left.N^{-1} \sum_{i=1}^{N} y_{i t}\right)$. Combining these results, we can rewrite the model (1) as

$$
y_{i t}=\beta^{\prime} \mathbf{x}_{i t}+\delta^{\prime} \mathbf{s}_{i t}+\lambda_{i}^{\prime} \mathbf{w}_{t}+\alpha_{i}^{*}+v_{i t}^{*}
$$

where $\alpha_{i}^{*}=-\alpha_{i}+\frac{\varphi_{i}}{\bar{\varphi}} \bar{\alpha}, v_{i t}^{*}=v_{i t}+\frac{-\varphi_{i}}{\bar{\varphi}} \bar{v}_{t}, \mathbf{w}_{t}=\left(\bar{y}_{t}, \overline{\mathbf{x}}_{t}^{\prime}, \overline{\mathbf{s}}_{t}^{\prime}, t\right)^{\prime}$, and $\lambda_{i}=\left(\lambda_{1 i}, \lambda_{2 i}^{\prime}, \lambda_{3 i}^{\prime}, \lambda_{4 i}\right)^{\prime}$ with $\lambda_{1 i}=\frac{\varphi_{i}}{\bar{\varphi}}, \lambda_{2 i}=-\lambda_{1 i} \beta, \lambda_{3 i}=-\lambda_{1 i} \delta$ and $\lambda_{4 i}=\lambda_{1 i} \bar{b}-b_{i}$. Provided that the

\footnotetext{
${ }^{3}$ Factor models are shown to exhibit the strong cross-sectional dependence since the maximum eigenvalue of the covariance matrix for $\varepsilon_{i t}$ tends to infinity at rate $N$ as $N \rightarrow \infty$, whilst other models such as spatial AR or MA models display much lower degrees of cross-sectional dependence, see Chudik et al. (2011) for details.

${ }^{4}$ Bai (2009) examines the cross-section dependence in panels more extensively, and allows regressors to be correlated with both factors and loadings by including both additive and interactive fixed effects. He then proposes an estimation method in which the unobservable common factors can be consistently estimated by the principal components. In this regard, the extension of the current study using the Bai's approach will be an interesting future study since the successful implementation will shed further lights on capturing further dynamics even in the presence of weakly correlated idiosyncratic errors, $v_{i t}$.
} 
model given by (1) and (2) is asymptotically equivalent to the augmented model, (6) (i.e. $\bar{v}_{t} \rightarrow_{q m} 0$ as $N \rightarrow \infty$, where $\rightarrow_{q m}$ means convergence in quadratic mean), then technical inefficiency, $e_{i t}$ in (3) can be approximated by

$$
e_{i t} \simeq \max _{i}\left(\alpha_{i}^{*}+\lambda_{i}^{\prime} \mathbf{w}_{t}\right)-\left(\alpha_{i}^{*}+\lambda_{i}^{\prime} \mathbf{w}_{t}\right), t=1, \ldots, T .
$$

This approach has a couple of advantages over the existing approaches modelling the impacts of external factors on the stochastic frontier, which assume that the external factors affect the shape or the structure of the technology and that they directly influence the degree of technical inefficiency with which inputs are converted into outputs (Kumbhakar and Lovell, 2000). It is clear from (6) and (7) that the global variables affect the frontier through $\delta^{\prime} s_{i t}$, and technical inefficiency (movement towards or away from the frontier) through $\lambda_{3 i}^{\prime} \bar{s}_{t}$. Hence, by explicitly controlling for both individual global variables $\left(\mathbf{s}_{i t}\right)$ and augmenting unobserved factors $\left(\theta_{t}\right)$ with their cross-section averages $\left(\bar{s}_{t}=N^{-1} \sum_{i=1}^{N} s_{i t}\right)$, our approach can capture the impacts of global shocks such as openness and trade policy on the production frontier and technical efficiency, simultaneously.

To obtain the consistent estimate of $e_{i t}$ in (7), we need to derive consistent estimates of heterogeneous parameters, $\alpha_{i}^{*}$ and $\lambda_{i}$ for $i=1, \ldots, N$. Replacing $\beta$ by $\hat{\beta}_{P C C E}$ and $\delta$ by $\hat{\delta}_{P C C E}$ in (6) and rearranging the result, we obtain:

$$
\tilde{y}_{i t}=\alpha_{i}^{*}+\lambda_{i}^{\prime} \mathbf{w}_{t}+\tilde{v}_{i t}, i=1, \ldots, N, t=1, \ldots, T,
$$

where $\tilde{y}_{i t}=y_{i t}-\hat{\beta}_{P C C E}^{\prime} \mathbf{x}_{i t}-\hat{\delta}_{P C C E}^{\prime} \mathbf{s}_{i t}$ and $\tilde{v}_{i t}=v_{i t}^{*}-\left(\hat{\beta}_{P C C E}-\beta\right) \mathbf{x}_{i t}-\left(\hat{\delta}_{P C C E}^{\prime}-\delta\right) \mathbf{s}_{i t}$ $=v_{i t}^{*}+o_{p}(1)=v_{i t}+o_{p}(1)$. For sufficiently large $T$, it is easily seen that $\alpha_{i}^{*}$ and $\lambda_{i}$ can be consistently estimated by the OLS estimators, denoted $\hat{\alpha}_{i}^{*}$ and $\hat{\lambda}_{i}$ respectively, by running the regression of (8) separately for each country. ${ }^{5}$ Hence, the time-varying individual and common technical inefficiencies can be consistently estimated by

$$
\hat{e}_{i t}=\max _{i}\left(\hat{\alpha}_{i}^{*}+\hat{\lambda}_{i}^{\prime} \mathbf{w}_{t}\right)-\left(\hat{\alpha}_{i}^{*}+\hat{\lambda}_{i}^{\prime} \mathbf{w}_{t}\right), t=1, \ldots, T .
$$

Then, the consistent estimates of the time-varying individual and common technical efficiencies, denoted $\tau_{i t}$ and $\tau_{t}$, can be obtained by ${ }^{6}$

$$
\hat{\tau}_{i t}=\exp \left(-\hat{e}_{i t}\right) \text { and } \overline{\hat{\tau}}_{t}=\exp \left(N^{-1} \sum_{i=1}^{N} \hat{e}_{i t}\right), t=1, \ldots, T \text {. }
$$

\footnotetext{
${ }^{5}$ Monte Carlos studies by Serlenga and Shin (2007b) confirm that the biases of the PCCE estimators of slope parameters (i.e. $\beta$ and $\delta$ ) decrease with $N$ and $T$. We have also conducted further Monte Carlo studies, showing that the biases of $\alpha_{i}^{*}$ also tend to decrease with $N$ and $T$. For example, we find that even when $(N, T)=(25,25)$, the biases of all the estimators used in the current study are reasonably small. These results are available upon request.

${ }^{6}$ In the empirical section below the common efficiency, $\overline{\hat{\tau}}_{t}$, is calculated as a weighted average where the weights are given by the share of GDP.
} 


\subsection{Dynamic Analysis of Common Efficiency and Globali- sation Factors}

To further investigate dynamic interactions between time-varying common EU efficiencies and global factors, we follow Borensztein et al. (1998) and Cameron et al. (2005), and focus on FDI and total imports as two main globalisation factors. Then, we consider the $\operatorname{VAR}(p)$ model:

$$
\mathbf{z}_{t}=\alpha+\sum_{j=1}^{p} \boldsymbol{\Phi}_{j} \mathbf{z}_{t-j}+\epsilon_{t}, \epsilon_{t} \sim i i d(\mathbf{0}, \mathbf{\Sigma}),
$$

where $\mathbf{z}_{t}=\left(\overline{\mathbf{g}}_{t}^{\prime}, \overline{\hat{\tau}}_{t}\right)^{\prime}, \overline{\mathbf{g}}_{t}$ is an $(m-1) \times 1$ vector of common globalisation factors, $\overline{\hat{\tau}}_{t}$ the common efficiency given by (10), $\alpha$ an $m \times 1$ vector of intercepts, $\boldsymbol{\Phi}_{i}$ an $m \times m$ matrix of coefficients, and $p$ the lag order. It is assumed that $E\left(\epsilon_{i t}\right)=\mathbf{0}$ and $E\left(\epsilon_{i t} \epsilon_{i s}^{\prime}\right)=\Sigma$ for $t=s$ with $\Sigma$ being an $m \times m$ positive definite matrix.

\subsubsection{Spectral analysis}

We briefly describe how the relative importance of efficiency cycle can be analysed using the spectral analysis. The bivariate spectrum of two stationary series, $x_{t}$ and $y_{t}$, is defined as the Fourier transform of the covariance function, $\boldsymbol{\Gamma}_{x y}$ :

$$
\mathbf{F}_{x y}(\omega)=\frac{1}{2 \pi} \sum_{\tau=-\infty}^{\infty} \boldsymbol{\Gamma}_{x y}(\tau) e^{-i \omega \tau}, \omega \in[-\pi, \pi],
$$

where the off-diagonal cross-spectrum at frequency $\omega$ is given by

$$
f_{x y}(\omega)=c_{x y}(\omega)-i q_{x y}(\omega), \omega \in[-\pi, \pi],
$$

where $c_{x y}(\omega)$ is the co-spectrum measuring a covariance between "in-phase" components of $x_{t}$ and $y_{t}$, and $q_{x y}(\omega)$ the quadrature spectrum, measuring a covariance between their "out-of-phase" components. The squared coherency, measuring the degree of a linear relationship between $x_{t}$ and $y_{t}$ at each frequency, is obtained by

$$
s c(\omega)=\frac{\left|f_{x y}(\omega)\right|^{2}}{f_{x}(\omega) f_{y}(\omega)}, \quad 0 \leq s c(\omega) \leq 1 .
$$

The variance of cyclical components of $y_{t}$ in a frequency band $\left[\omega_{1}, \omega_{2}\right]$ is decomposed into explained and unexplained parts:

$$
\int_{\omega_{1}}^{\omega_{2}} f_{y}(\omega) d \omega=\int_{\omega_{1}}^{\omega_{2}} s c(\omega) f_{x}(\omega) d \omega+\int_{\omega_{1}}^{\omega_{2}} f_{u}(\omega) d \omega
$$


where $f_{y}(\cdot), f_{x}(\cdot)$, and $f_{u}(\cdot)$ are the autospectra of $y, x$ and $u$, respectively. This enables us to compare the degree of a linear relationship between cycles of different series. Further, the explained variance can be decomposed into in-phase and outof-phase components:

$$
\int_{\omega_{1}}^{\omega_{2}} s c(\omega) f_{x}(\omega) d \omega=\int_{\omega_{1}}^{\omega_{2}} \frac{\left[c_{x y}(\omega)\right]^{2}}{f_{x}(\omega) f_{y}(\omega)} f_{x}(\omega) d \omega+\int_{\omega_{1}}^{\omega_{2}} \frac{\left[q_{x y}(\omega)\right]^{2}}{f_{x}(\omega) f_{y}(\omega)} f_{x}(\omega) d \omega
$$

where $\left|f_{x y}(\omega)\right|^{2}=\left[c_{x y}(\omega)\right]^{2}+\left[q_{x y}(\omega)\right]^{2}$ (Mastromarco and Woitek 2007). Notice that information on co-movement or synchronisation is provided by the in-phase component of explained variance.

From the $\operatorname{VAR}(p)$ model, (11), we obtain an $m \times m$ spectral density matrix by

$$
\mathbf{F}_{\mathbf{z}}(\omega)=\frac{1}{2 \pi} \boldsymbol{\Phi}(\omega)^{-1} \boldsymbol{\Sigma} \boldsymbol{\Phi}(\omega)^{-1 *}, \omega \in[-\pi, \pi]
$$

where $\boldsymbol{\Phi}(\omega)$ is the Fourier transform of $\boldsymbol{\Phi}(L)=\mathbf{I}_{m}-\sum_{j=1}^{p} \boldsymbol{\Phi}_{j} L^{j}$, and '*' denotes the complex conjugate transpose. We then derive the cross-spectra and the inphase explained variance. This enables us to evaluate the extent to which cycles of globalisation factors and those of common efficiency co-move and synchronise.

\section{Empirical Results}

The first part of our empirical analysis is conducted using the (augmented) stochastic frontier model as described in Subsection 2.1. This approach is linked to the growth accounting literature, ${ }^{7}$ but more flexible by distinguishing between the effects of globalisation variables on the production technology (shift of the frontier) and on efficiency (movement away/towards the frontier). Within the SF framework there has been relative silence on the issue of dynamic adjustments of efficiency in conjunction with factors, which is mainly due to their possible endogeneity. This issue can be explicitly addressed within the second-stage VAR framework where efficiency and globalisation factors are modelled simultaneously. By analysing the flexible dynamic interactions between efficiency and globalisation factors, we aim to contribute to agnostic empirical evidence on the issue whether globalisation and efficiency gains can be mutually determined.

\footnotetext{
${ }^{7}$ Barro and Sala-i-Martin (2004) identify the Solow residuals as technical changes, though they represent a total factor productivity capturing both technological and efficiency changes. Alternatively, Caves et al. (1982) propose the Malmquist productivity index, defined as a distance function in order to distinguish between technological changes and efficiency changes.
} 


\subsection{The Data}

The data is collected for 18 countries of the EU (Austria, Belgium, Denmark, Finland, France, Germany, Greece, Iceland, Ireland, Italy, Luxembourg, the Netherlands, Norway, Portugal, Spain, Sweden, Switzerland and the UK) over the period, 1970-2004 (35 years). GDP is measured in million US dollars at the 2000 price and labour measured as total employment in thousands. Capital is measured in millions US dollars at 2000 and constructed using the perpetual inventory method $(\mathrm{PIM}) .^{8}$ All three variables are logged before estimation. For globalisation factors we identify two most important channels: total imports and FDI inflows (measured as net inflows of foreign direct investment) which are both transformed as a ratio to GDP. Global factors are constructed as the weighted averages where the weights are given by the share of GDP. Capital is sourced from PWT 6.2; labour from OECD Labour Force Statistics; GDP, imports and FDI from the World Bank World Development Indicators and Unctad. The sample period is selected by the data availability. FDI and imports include both intra-EU and extra-EU country flows such that any policy recommendation will refer to the EU policy towards the global economy. For example, the protective common agriculture policy (CAP) in the EU can be regarded as a system of import tariffs and subsidies to the EU farmers.

By focussing on OECD EU countries we can maintain the key assumption of SFA that the same technology is available to all similar countries. This also enables us to overcome the limitations of several cross-country studies that assume equal quality of production factors such as level of education, skills and depreciation rates. Hence, without loss of generality, the distance between a country's frontier and the best practice frontier can represent inefficiency of the particular country. Productivity growth in Europe has been declining since the mid 90s and the fiscal policy coordination embedded in the Growth and Stability Pact in 1993 failed to boost growth. Thus, the Lisbon agenda, launched in 2000, aims to make the EU more competitive in a globally integrated environment by pressing the member countries to liberalise their product and labor markets and promote technology innovations. Notice that some progresses have been achieved in trade and goods markets whilst service sectors still remain full of impediments to free

\footnotetext{
${ }^{8} \mathrm{PIM}$ is necessitated by the lack of capital stock data across all the countries. For an individual country, the capital stock is constructed as $K_{t}=K_{t-1}(1-\theta)+I_{t}$, where $I_{t}$ is investment and $\theta$ the rate of depreciation assumed to be $6 \%$ (e.g., Hall and Jones, 1999; Iyer et al., 2008). Repair and maintenance are assumed to keep the physical production capabilities of an asset constant during its lifetime. Initial capital stocks are constructed, assuming that capital and output grow at the same rate. Specifically, for country with investment data beginning in 1970, we set the initial stock, $K_{1970}=I_{1969} /(g+\theta)$, where $g$ is the average 10-year output growth rate from 1970 to 1980 and $I$ is investment in gross physical capital stock. Estimated capital stock includes both residential and nonresidential capital.
} 
market competition.

Descriptive statistics summarised in Table 1 provide a number of stylised findings. Firstly, import is much more volatile than FDI. Secondly, smaller economies such as Belgium, Ireland, Luxembourg, Netherlands display the higher propensity to trade and to attract FDI. ${ }^{9}$ Finally, the second and the third panel in Figure 1 show that growth rates of both FDI and imports peak in 2000 and then decrease (rather rapidly for FDI). ${ }^{10}$

[Table 1 about here]

\subsection{Stochastic Frontier and Technical Efficiency}

Table 2 summarises the estimation results for the stochastic frontier model, (1) and (2) by alternative estimations; namely, Pooled OLS (POLS), Fixed Effect (FE) and PCCE estimators. Both labour and capital elasticities are all statistically significant. Moreover, labour's contributions are significantly higher as expected, implying that it is easier to maintain output and profitability by reducing employment or increasing labour productivity rather than by dismissing capital stocks.

POLS and FE estimates are too high, suggesting that both estimates are biased upward. These biases might be caused by either neglected cross-section error dependency (Pesaran, 2006) or misspecification of individual effects when they are time-varying (Bai, 2009). In particular, we expect that cross-section dependency is highly likely to be present in our sample of the EU countries, and thus apply the diagnostic test for cross-section dependency advanced by Pesaran (2004). We find that the null of cross-section independence is strongly rejected at the $1 \%$ level. Hence, this result supports that the PCCE provides more reliable estimates of labor and capital elasticities respectively at 0.51 and $0.44 .{ }^{11}$ Furthermore, as confirmed by an insignificant t-ratio for the null of the sum of elasticities being

\footnotetext{
${ }^{9}$ Luxembourg is both the largest recipient of FDI and largest trade oriented due to "transshipped' FDI, i.e. how companies transfer funds between affiliates within the same group located in different countries, or channel funds to acquire companies in different countries through a holding company. For these reasons, Luxembourg is often treated as an outlier, e.g. Edwards (1998) and Daniels et al. (2005). We have also estimated the model without including Luxembourg but obtained qualitatively similar results.

${ }^{10}$ The peak in 2000 was argued to be mainly driven by a large amount of shares and acquisition operations spearheaded by rapid information technological changes during the 1990s mostly through the regulatory relaxation, as also reported in official statistics in World Bank, Eurostat and Unctad.

${ }^{11}$ Staring from the full set of the augmented factors, $\mathbf{w}_{t}=\left(\bar{y}_{t}, \bar{l}_{t}, \bar{k}_{t}, \overline{F D I}_{t}, \bar{M}_{t}, t\right)^{\prime}$ in $(6)$, we have estimated the model with various combinations of augmented factors, and then selected the final empirical specification (their estimations results reported in Table 2) on the basis of overall statistical significance and parsimony. Overall estimation results are qualitatively similar across different specifications.
} 
equal to unity, our finding supports the widely accepted empirical evidence that technology in the EU follows the constant returns to capital and labour. ${ }^{12}$ We also find that the impacts of imports and FDI on the frontier are both significantly positive, with the imports having a relatively greater influence.

[Table 2 about here]

Next, on the basis of the PCCE estimation results, we continue to follow the estimation procedure as described in (9)-(10), and obtain consistent estimates of individual and common efficiency measures, denoted $\hat{\tau}_{i t}$ and $\overline{\hat{\tau}}_{t}$, respectively. Notice that our approach can control for the effects of globalisation variables on production structure and efficiency separately as described in Subsection 2.1. In particular, this decomposition enables us to identify the efficiency changes (movement towards or away from the frontier) related to globalisation factors.

Table 3 summarises descriptive statistics for individual efficiency for $18 \mathrm{EU}$ countries. We find that the most efficient countries over the sample period are the UK, Germany and France while the least efficient are Iceland, Luxembourg and Portugal. A detailed analysis of individual technical efficiency $\left(\hat{\tau}_{i t}\right)$ reveals that Finland, Ireland, Luxembourg, Sweden and the UK have maintained positive growth rates, mainly due to an increased investment in information and technology communication (ITC) sector and an implementation of policies in favor of openness. The Netherlands have also registered an increase in TFP, especially in the service sector. Interestingly, poor efficiency growth of Germany is closely related to its unification process. Germany registered a stable (though declining) efficiency growth before unification, achieved a peak after unification and experienced a slowdown after 1995, that was partially caused by a post-unification expansionary fiscal policy. Negative performance is also observed in Austria and Denmark where the spill-over effects of ITC investment did not seem to spread. Switzerland has also underwent a slowdown in efficiency growth especially in the service sector. Finally, France and Spain have displayed quite a similar pattern of slow efficiency growth largely due to market rigidities. Overall, this (heterogeneous) evidence may suggest that efficiency slowdowns are likely to be closely related to market rigidities and imperfections. Theoretically, Helpman and Itskhoki (2007) develop a model for analysing the role of labor market rigidities and trade impediments in shaping welfare and productivity, and show that a flexible country with lower labor market frictions has a higher TFP. Further, Aghion et al. (2009) provide an empirical evidence on significant negative effects of market and labour rigidities on TFP growth.

\footnotetext{
${ }^{12}$ The hypothesis of the constant return to scale turns out to be suitable for industrialised countries (e.g. Malley et al., 2005; Iyer et al., 2008). Further, we cannot reject the null hypothesis that the production function is of the Cobb-Douglas form against the translog form.
} 
Overall, the results reported in Table 3 are remarkably consistent with growth patterns of multifactor and labour productivity in the EU over the period 1970 - 2004, as documented in the EU KLEMS Productivity Report (Van Ark et al., 2007). Furthermore, we also conduct the subperiod (1970-1995 and 1996-2004) analysis, and find that efficiency growth starts to slow down from the mid 90s. This pattern is generally consistent with a number of OECD reports (e.g. Eichengreen, 2004), and will be confirmed at a global level as analysed below.

[Table 3 about here]

\subsection{Dynamic Analysis of Common Efficiency and Factors}

We now examine dynamic interactions between the EU common time-varying efficiencies $\left(\overline{\hat{\tau}}_{t}\right)$, and two common global factors, imports and FDI. After complying with the usual procedure for determining the lag order and checking for stability conditions, we fit the trivariate $\operatorname{VAR}(2)$ model: ${ }^{13}$

$$
\mathbf{z}_{t}=\alpha+\boldsymbol{\Phi}_{1} \mathbf{z}_{t-1}+\boldsymbol{\Phi}_{2} \mathbf{z}_{t-2}+\varepsilon_{t},
$$

where $\mathbf{z}_{t}=\left(\overline{F D I}_{t}, \bar{M}_{t}, \overline{\hat{\tau}}_{t}\right)^{\prime}$ and $\overline{F D I}_{t}, \bar{M}_{t}$ are the cross-section weighted averages of the ratios of FDI and imports to GDP.

Figure 1 provides time-varying patterns of level and growth rates of $\overline{\hat{\tau}}_{t}, \overline{F D I}_{t}$ and $\bar{M}_{t}$. Similar to the results of the individual efficiency as described above, the EU has undergone a marked slowdown in efficiency growth from the mid 1990s (growth rates are $10.6 \%$ for 1970-1995 and 1.6\% for 1996-2004). FDI and total imports grow by $25 \%$ and $45 \%$, respectively, over the whole period. Over the second subperiod, 1996-2004, however, imports grow by $20 \%$ but FDI declines by $30 \%$ even though FDI registered a peak in 1999-2000. This descriptive analysis clearly provides some preliminary insights on the sources of the (time-varying) EU productivity, suggesting that the recent slowdown of both productivity and FDI are closely related (e.g. Busse and Groizard, 2006). In particular, the crises that hit telecommunications and capital intensive fields such as oil, gas production and banking after 2000, appear to account mostly for declining FDI flows and productivity, as documented in Van Ark et al. (2007) and Taylor (2008).

\footnotetext{
${ }^{13}$ To save space we do not report the estimation results of (16), which do not suffer from any serious misspecification. Though we mainly focus on modelling the dynamic interactions between efficiency and openness factors simultaneously, we have also conducted the Granger causality tests for the following null hypotheses: (i) efficiency does not Granger-cause imports; (ii) efficiency does not Granger-cause FDI; (iii) imports do not Granger-cause efficiency; (iv) FDI does not Granger cause efficiency. We find that the $p$-values of the $\chi^{2}$ tests are $0.079,0.334$, 0.001, and 0.029, respectively. Combined together, we may conclude that the causation is likely to run from imports and FDI to efficiency. This evidence will provide further support for our focus on the impulse responses of efficiency with respect to imports and FDI shocks below.
} 
[Figure 1 about here]

Based on the VAR estimation results, we extrapolate two pieces of evidence on synchronization of efficiency and openness cycles, and the time delayed effects on efficiency (technological catch-up) of shocks to openness factors.

Spectral analysis of efficiency and globalisation factors It is generally accepted that the recent decline in the EU productivity has largely resulted from weak growth in TFP. Slow technological catch-up often causes the lack of convergence in output levels (e.g. Mankiw et al., 1992; Barro and Sala-i-Martin, 2004). An increase in efficiency represents movement towards the frontier, though it does not necessarily imply that technology transfer will reduce the gap between outperforming and underperforming countries, since the former may benefit from efficiency improvements as much as or even more than the latter.

To better evaluate the technological catching-up process of the EU, we turn to synchronisation of the business cycles of $\overline{\hat{\tau}}_{t}, \overline{F D I}_{t}$ and $\bar{M}_{t}$. To identify the relative importance of the relationship between these components over 3 to 10 years, we evaluate the spectra of the VAR model, (16), and derive the in-phase component of explained variance as a measure of synchronisation (Mastromarco and Woitek, 2007). Table 4 reports the proportion of the share of total variances, explained variances and in-phase explained variances in the frequency bands, i.e. the cycles with a length of 3-5 years (the Kitchin cycle), 5-7 years and 7-10 years (the Jugular cycle). We find that common efficiency is dominated by the shorter cycle of 3-5 years, and weakly co-moves with both imports and FDI. Notice that such weak co-movements with FDI can be easily explained by the adjustment costs involved in FDI, e.g. Tybout (1992) and Coe and Helpman (1995).

\section{[Table 4 about here]}

Figure 2 displays the spectral measures; the first line is the variance of common efficiency at each frequency, the second the explained variance of common imports and FDI, and the third the explained variance in phase. The dominant cycle of common efficiency is estimated at frequency 0.27 , corresponding to the cycle of about 4 years (autospectrum in blue line). ${ }^{14}$ The dominant frequencies contain important information of the structure of efficiency. To find how strong is the inter-relationship between efficiency and common imports and FDI, we turn to the squared coherency (the explained variance, a measure similar to $R^{2}$ ) in the

\footnotetext{
${ }^{14}$ Notice that the spectrum is expressed as a function of radians, $\omega=2 \pi \lambda$ with $\lambda \in[-0.5,0.5]$ rather than time units, see Priestley (1981).
} 
second (green) line, ${ }^{15}$ which measures the proportion of common efficiency variance over a particular interval attributable to $\bar{M}_{t}$ and $\overline{F D I}_{t}$, respectively. Now, the cycle range between 3-5 years dominates the relationship (see also Table 4). To further analyse the co-movement, we turn to the last (red) line representing the "inphase" component of explained variance, that measures the extent to which the two series reach upper and lower turning points at the same time. Common efficiency fluctuations are more closely related to FDI cycles than imports cycles at most frequencies. In particular, the in-phase component of explained variance with FDI is higher at cycles of 5-7 years and 7-10 years (see also Table 4), providing evidence that the positive correlation between the FDI shocks and efficiency become higher over the medium- to the long-term. ${ }^{16}$

[Figure 2 about here]

Dynamic transmission channels between common factors and efficiency Figure 3 displays the orthogonalised and cumulated impulse response function (IRF) of $\overline{\hat{\tau}}_{t}$ with respect to one standard deviation shock to each of the $\bar{M}_{t}$ and $\overline{F D I}_{t}$ equations with the bootstrap-based confidence intervals. In order to facilitate the interpretation, we consider it prudent to normalise IRFs such that the effect of a shock to the $j$-th equation on the $j$-th variable is unity on impact. Thus, we can evaluate the effect on efficiency relative to $1 \%$ increase in FDI and import, respectively.

Figure 3(a) shows that IRFs of common EU efficiency to import shocks are all positive as expected, suggesting that positive import shocks are likely to play an important role in promoting efficiency. These IRFs reach a peak after 4 years at which $1 \%$ increase in import will raise efficiency by $0.02 \%$, and then approach zero monotonically as the horizon increases. Figure 3(b) also shows that cumulative IRFs of efficiency to (positive) import shocks are significantly positive up to the forecast horizon of 20 years. The IRFs of the EU efficiency to the FDI shocks in Figure 3(c) are mostly positive except for the initial three years. Interestingly, the impulse responses peak after 9 years at which $1 \%$ increase in FDI boosts efficiency by $0.03 \%$, and then slowly die out to zero. Cumulative IRFs of FDI in Figure 3(d) clearly show that FDI exert positive effects on efficiency especially in the mediumto the long-run (i.e. after 5 years). Overall impacts of FDI are slightly larger than those of imports (but not statistically significant) as reported in the long-run

\footnotetext{
${ }^{15}$ The partial squared coherency can be used to calculate the proportion of variance in a frequency band due to a specific variable, with the influence of other variables removed, see Koopmans (1974). This enables us to consider isolated effects of fluctuations in imports and FDI on common efficiency.

${ }^{16}$ Given the small time period observations of our data set (35 annual observations), we note in passing that cycles of 10 years or more cannot be accurately identified.
} 
cumulative IRFs (evaluated at the 40 year-horizon) of $0.2 \%$ for imports and $0.5 \%$ for FDI.

In sum, globalisation factors proxied by import and FDI may help the EU countries improve their efficiency position relative to the frontier. This may suggest that openness will be a vital factor in fostering the technology catch-up, thus providing support for the beneficiary implication of the global trade expansion as documented by Edwards (1998), Frankel and Romer (1999) and Alcalà and Ciccone (2004).

[Figure 3 about here]

Further comparison of the IRFs and cumulated IRFs in Figure 3 reveals an important transmission channel through which imports and FDI may spread efficiency across the EU countries; namely their different time delayed effects on technical efficiency. The impacts of import shocks on efficiency are much stronger in the short-run as also confirmed by the spectral analysis showing that the synchronisation between efficiency and imports are higher at the shorter cycles (see Table 4). On the other hand, positive impacts of FDI shocks on efficiency are realised much later and more persistent than those of import shocks, confirming that technology catch-up in response to FDI shocks generally requires a substantial time period to be fully transferred. In this regard, our result is in accordance with Cohen and Levinthal (1989), who observe that the competence to evaluate and utilise outside knowledge is largely a function of prior related knowledge. Earlier, we find that efficiency and FDI display the higher synchronisation at business cycle of 5-7 and 7-10 years and that the in-phase components of FDI in explained variance are higher than imports at all frequencies, especially at shorter frequencies (i.e. after 5 years). Hence, FDI seems to play a more important role in accelerating technology catching-up in the EU over the medium- to the long-term. This evidence is generally consistent with the sample observation that both productivity and FDI have started to slow down around 2000.

Further, globalisation factors can be closely related to internal and external demand and supply fluctuations. Demand and supply shocks are more likely to be transmitted across countries that are closely linked through openness channels (Krugman, 1991). Hence, a careful evaluation of whether FDI and trade are proor counter-cyclical with efficiency provides us an important policy implication. Combining the estimation results for spectral analyses and impulse response functions, we may conclude that imports are pro-cyclical only in the short-run whereas FDI are pro-cyclical mostly from the medium- to the long-run. An improvement in FDI and trade will be likely to enhance efficiency and promote business cycle synchronisation. This leads to an important policy suggestion especially under the current global recession period: the EU government's policy to encourage openness 
could help the member countries to faster exit a recession through the technology catch-up and the associated total factor productivity improvement, e.g. Wacziarg and Welch (2008) and ECB (2009). Hence, the protection-oriented policy will be more detrimental on technology catch-up in the EU and is likely to exert further negative impacts on the global economy.

\section{Conclusion}

This paper aims to address an important policy issue on whether there exists a transmission mechanism under which technological diffusion plays a significant role in spurring the productivity growth by lowering barriers to flows of foreign goods and investments. To this end we propose a two-step approach: in the first step we estimate the panel-based stochastic frontier model with factors, and obtain robust estimates of individual and common technical efficiency measures in the EU. Next, we apply a trivariate VAR approach to model dynamic interactions between common efficiency and two globalisation factors proxied by FDI and imports. In this regard, our proposed methodology enables us to investigate the influence of globalisation factors on the EU common efficiency in a fully dynamic setting.

Our main empirical findings are summarised as follows: Firstly, the recent slowdown of the EU productivity has been closely related to the corresponding FDI decline especially after 2000. Secondly, the impulse response analysis illustrates that the EU common efficiency is likely to be boosted by the FDI and import shocks, both of which act as the transmission channels to diffuse common technology. Furthermore, the impacts of import shocks are significantly higher in the short run while the FDI shocks become more significant over the medium- to the long-term, suggesting that the knowledge transfer associated with FDI requires a substantial time period to diffuse efficiency externalities. Finally, with the EU technical efficiency, imports are pro-cyclical only in the short-run while FDI are pro-cyclical from the medium- to the long-run.

It is worth mentioning some of the avenues for further research opened by this paper. A number of studies examine the role of firm dynamics and intra-firm productivity in the analysis of aggregate productivity growth, and highlight that neglecting these micro structure effects are likely to lead to aggregation bias, e.g. Balk (2003). Further, Garrett (2003) stresses that the degree of aggregation bias depends on the covariance between cross-section individual residuals. Though our approach may overcome this issue partially by allowing for cross-section dependence across countries, an extension of our framework to deal with this potentially important issue warrants further research. 
Table 1: Descriptive statistics for variables used in estimations

\begin{tabular}{lrrrrrr}
\hline \hline & & $\mathrm{y}$ & $\mathrm{k}$ & $\mathrm{l}$ & $M$ & $F D I$ \\
\hline AUT & mean & 25.65 & 15.79 & 8.11 & 35.67 & 0.85 \\
& $\mathrm{sd}$ & 0.25 & 0.28 & 0.09 & 4.89 & 0.97 \\
BEL & mean & 25.87 & 15.68 & 8.24 & 63.87 & 8.61 \\
& $\mathrm{sd}$ & 0.23 & 0.23 & 0.04 & 9.5 & 17.98 \\
DNK & mean & 25.52 & 15.93 & 7.84 & 34.57 & 1.28 \\
& $\mathrm{sd}$ & 0.19 & 0.15 & 0.05 & 3.35 & 2.01 \\
FIN & mean & 25.17 & 16.03 & 7.74 & 28.02 & 1.24 \\
& $\mathrm{sd}$ & 0.26 & 0.13 & 0.06 & 2.77 & 2.16 \\
FRA & mean & 27.6 & 15.77 & 10.02 & 21.87 & 1.13 \\
& $\mathrm{sd}$ & 0.24 & 0.22 & 0.05 & 3.03 & 0.99 \\
DEU & mean & 27.97 & 15.96 & 10.32 & 24.49 & 0.75 \\
& $\mathrm{sd}$ & 0.23 & 0.15 & 0.15 & 4.35 & 1.80 \\
GRC & mean & 25.2 & 15.49 & 8.19 & 25.8 & 0.79 \\
& $\mathrm{sd}$ & 0.22 & 0.1 & 0.1 & 4.11 & 0.36 \\
ISL & mean & 22.48 & 15.74 & 4.8 & 36.38 & 0.95 \\
& $\mathrm{sd}$ & 0.33 & 0.29 & 0.2 & 3.73 & 1.26 \\
IRL & mean & 24.54 & 15.13 & 7.12 & 57.99 & 3.93 \\
& $\mathrm{sd}$ & 0.5 & 0.34 & 0.17 & 11.46 & 6.87 \\
ITA & mean & 27.43 & 15.72 & 9.94 & 20.9 & 0.45 \\
& $\mathrm{sd}$ & 0.24 & 0.19 & 0.04 & 2.85 & 0.34 \\
LUX & mean & 23.11 & 16.06 & 5.23 & 91.6 & 17.61 \\
& $\mathrm{sd}$ & 0.43 & 0.31 & 0.23 & 16.5 & 1.39 \\
NLD & mean & 26.32 & 16.01 & 8.68 & 52.66 & 3.00 \\
& $\mathrm{sd}$ & 0.25 & 0.1 & 0.19 & 4.94 & 3.68 \\
NOR & mean & 25.41 & 16.12 & 7.59 & 34.68 & 1.15 \\
& $\mathrm{sd}$ & 0.34 & 0.21 & 0.11 & 4.35 & 1.09 \\
PRT & mean & 25.01 & 15.11 & 8.36 & 33.62 & 1.53 \\
& $\mathrm{sd}$ & 0.33 & 0.27 & 0.11 & 4.44 & 1.46 \\
ESP & mean & 26.7 & 15.36 & 9.49 & 20.3 & 1.74 \\
& $\mathrm{sd}$ & 0.28 & 0.27 & 0.11 & 5.47 & 1.48 \\
SWE & mean & 25.93 & 15.92 & 8.33 & 31.19 & 2.33 \\
& $\mathrm{sd}$ & 0.2 & 0.11 & 0.04 & 4.5 & 4.41 \\
CHE & mean & 26.02 & 16.4 & 8.18 & 33.35 & 0.62 \\
& $\mathrm{sd}$ & 0.15 & 0.12 & 0.12 & 3.42 & 2.28 \\
& $\mathrm{sd}$ & 0.23 & 0.25 & 0.04 & 2.56 & 1.59 \\
\hline & & & & & &
\end{tabular}

Notes: $y, k$ and $l$ denote logarithm of output, capital and labour. $M$ and $F D I$ are imports and FDI inflows measured as ratios of $G D P$. 
Table 2: Panel Data Estimation Results for Stochastic Frontier

\begin{tabular}{lrrrr}
\hline \hline & $\beta_{\text {capital }}$ & $\beta_{\text {labour }}$ & $\delta_{M}$ & $\delta_{F D I}$ \\
\hline POLS & $0.66^{*}$ & $1.002^{*}$ & $0.15^{*}$ & $0.03^{*}$ \\
& $(0.02)$ & $(0.006)$ & $(0.02)$ & $(0.006)$ \\
FE & $0.93^{*}$ & $0.57^{*}$ & $0.05^{*}$ & $0.02^{*}$ \\
& $(0.02)$ & $(0.03)$ & $(0.02)$ & $(0.003)$ \\
PCCE & $0.44^{*}$ & $0.51^{*}$ & $0.08^{*}$ & $0.01^{*}$ \\
& $(0.18)$ & $(0.09)$ & $(0.03)$ & $(0.004)$ \\
\hline \hline$t_{\text {crs }}$ & -0.21 & $p$-value 0.83 & & \\
$C D$ & 13.1 & $p$-value 0.00 & & \\
\hline
\end{tabular}

Notes: The estimates reported are coefficients of elasticity. The PCCE estimates have been performed using the following sets of observed and unobserved factors: unobserved factor $\left(\bar{k}_{t}\right)$ and observed factors $\left(\bar{s}_{t}=M, F D I\right)$. $t_{c r s}$ denotes t-test for the hypothesis of constant return to scale in the case of PCCE estimates; $C D$ denotes the general diagnostic test for cross-section dependency described in Pesaran (2004); * denotes significance at 1 per cent level; standard errors in parenthesis. 
Table 3: Descriptive statistics for efficiency

\begin{tabular}{lrrrr}
\hline \hline country & efficiency & $\begin{array}{r}\text { growth rate } \\
\text { 1970-2004 }\end{array}$ & $\begin{array}{r}\text { growth rate } \\
1970-1995\end{array}$ & $\begin{array}{r}\text { growth reate } \\
1996-2004\end{array}$ \\
\hline AUT & 0.306 & -0.004 & 0.020 & -0.026 \\
BEL & 0.351 & 0.141 & 0.520 & -0.234 \\
DNK & 0.289 & -0.017 & -0.030 & -0.004 \\
FIN & 0.211 & 0.971 & 1.430 & 0.508 \\
FRA & 0.853 & 0.221 & 0.550 & -0.108 \\
DEU & 0.967 & 0.109 & 0.400 & -0.185 \\
GRC & 0.22 & 0.536 & 0.370 & 0.698 \\
ISL & 0.072 & 0.351 & 0.540 & 0.160 \\
IRL & 0.217 & 1.511 & 1.850 & 1.167 \\
ITA & 0.775 & 0.515 & 1.230 & -0.196 \\
LUX & 0.084 & 0.506 & 1.030 & -0.021 \\
NLD & 0.388 & 0.287 & 0.440 & 0.130 \\
NOR & 0.271 & 1.079 & 1.570 & 0.590 \\
PRT & 0.193 & 0.554 & 1.220 & -0.109 \\
ESP & 0.55 & 0.217 & 0.760 & -0.325 \\
SWE & 0.347 & 0.256 & 0.260 & 0.253 \\
CHE & 0.326 & -0.528 & -0.680 & -0.372 \\
GBR & 1 & 0.000 & 0.000 & 0.000 \\
\hline
\end{tabular}

Notes: First column shows the mean value of efficiency for each country over the whole sample period. The second column presents average growth rates of efficiency calculated over the whole period - expressed as a percentage - ; the third and the fourth column exhibit average efficiency growth rates over the two subperiods 1970-1995 and 1996-2004 - expressed as a percentage - , respectively. 
Table 4: Spectral analysis of common efficiency $\left(\overline{\hat{\tau}}_{t}\right)$ and global factors $\left(\overline{F D I}_{t}\right.$ and $\left.\bar{M}_{t}\right)$

\begin{tabular}{lrrr}
\hline \hline & 7-10 years & $5-7$ years & $3-5$ years \\
\hline share of total variance & 0.045 & 0.142 & 0.694 \\
$\bar{M}_{t}$ & & & \\
explained & & & \\
in-phase & 0.004 & 0.033 & 0.281 \\
& 0.003 & 0.030 & 0.182 \\
$\overline{F D I}_{t}$ & & & \\
explained & & & \\
in-phase & 0.014 & 0.034 & 0.236 \\
\hline
\end{tabular}

Notes: The row on 'share of total variance' reports the estimated efficiency variance shares over each frequency range. The row on 'explained' presents the fraction of the variance shares that can be explained by the cycles of either imports or FDI while the row on 'in-phase' reports the in-phase component associated with the cycles of either imports or FDI. 
Figure 1: Time-varying common efficiency $\left(\overline{\hat{\tau}}_{t}\right)$ and global factors $\left(\overline{F D I}_{t}\right.$ and $\left.\bar{M}_{t}\right)$
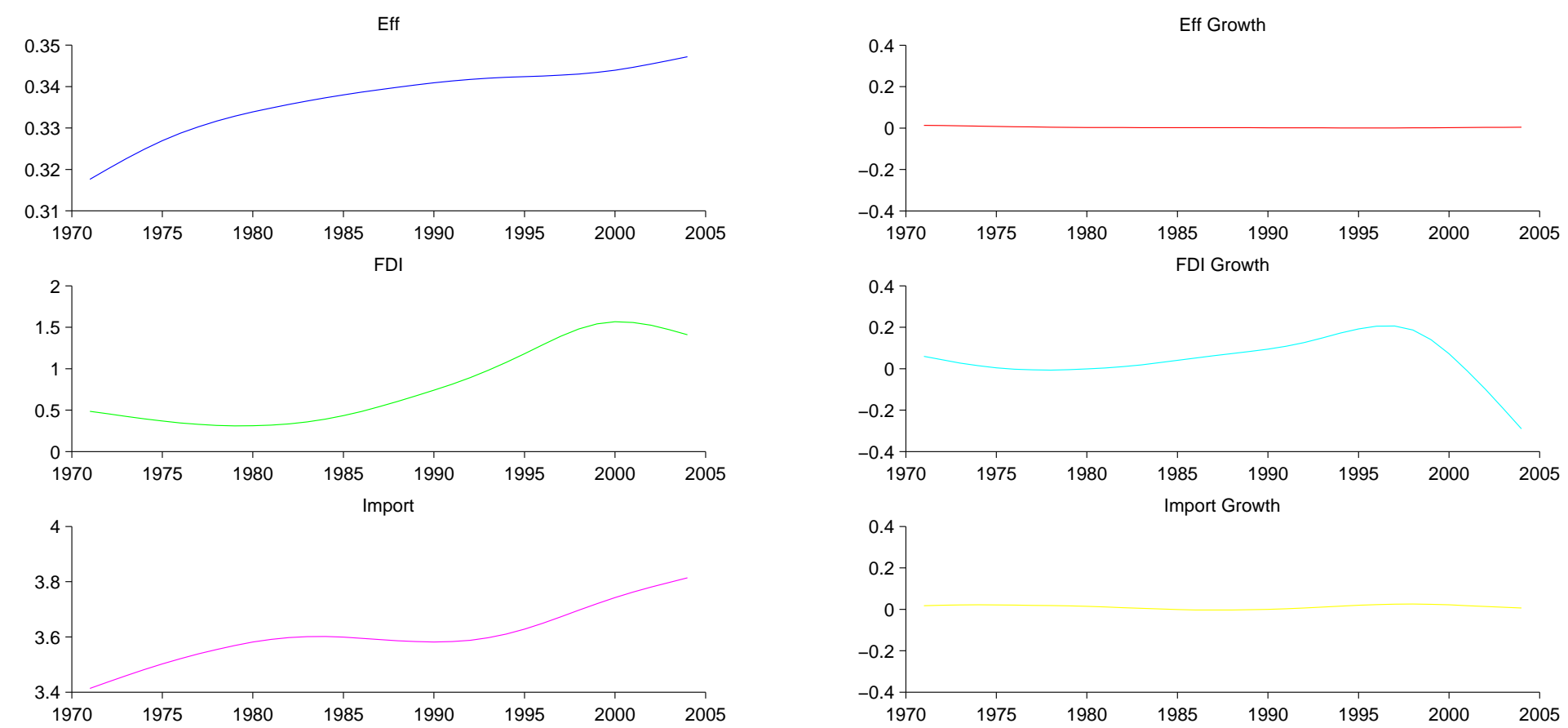

Notes: The first panel plots the (weighted) common efficiency, $\overline{\hat{\tau}}_{t}$, in level and growth rate. The second and the third panels display the weighted FDI and import factors in level and growth rate, respectively. Notice also that we use the Hodrick and Prescott (1997) filter to smooth the time paths with a smoothing weight, 100 . 
Figure 2: Comovement of business cycles of common efficiency $\left(\overline{\hat{\tau}}_{t}\right)$ and global factors $\left(\overline{F D I}_{t}\right.$ and $\left.\bar{M}_{t}\right)$
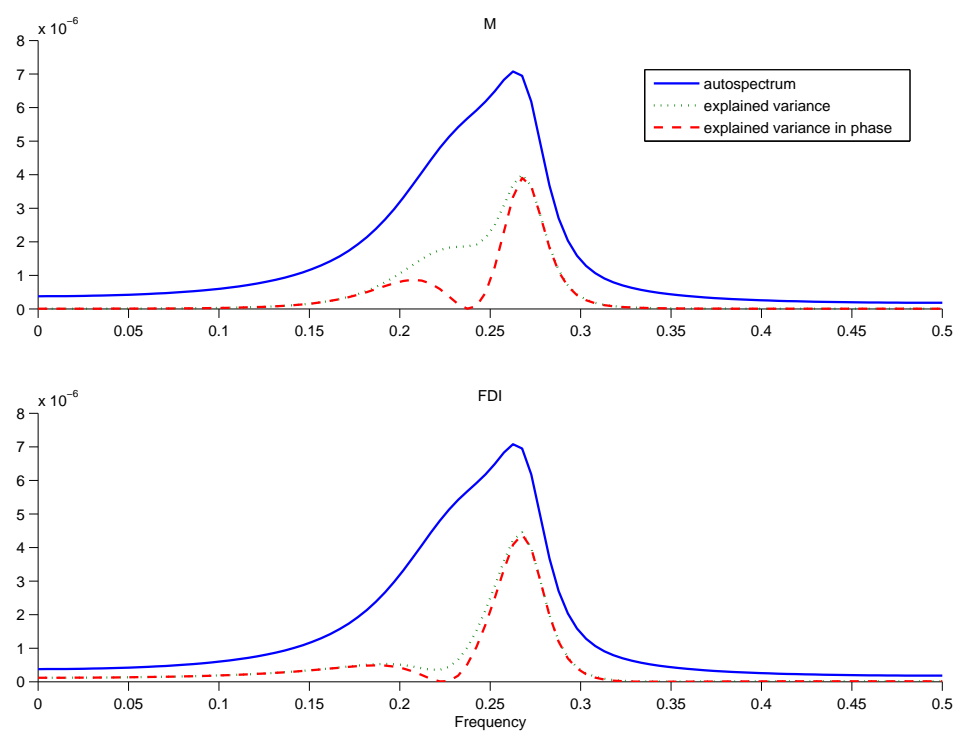

Notes: The blue lines (autospectrum) are the variance of common efficiency at each frequency. The green and the red lines indicate the explained variance and the explained variances in-phase of $\bar{M}_{t}$ and $\overline{F D I}_{t}$, respectively. 
Figure 3: Impulse response functions of common efficiency $\left(\overline{\hat{\tau}}_{t}\right)$
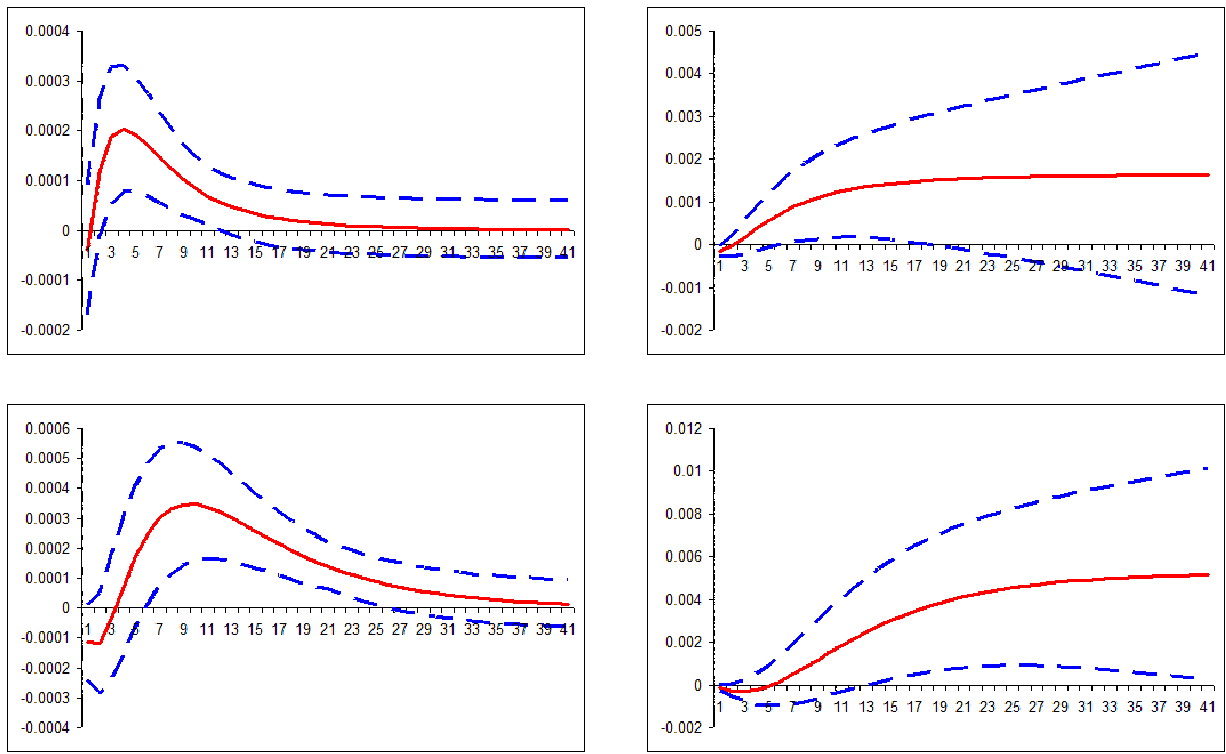

Notes: The graphs at the top panel display ortohogonalised and cumulative impulse response functions of efficiency with respect to one standard deviation shock to the $\bar{M}$ equation while the graphs on the bottom panel show ortohogonalised and cumulative impulse response functions of efficiency with respect one standard deviation shock to the $\overline{F D I}$ equation. All the results are obtained by estimating the $\operatorname{VAR}(2)$ model for $(\overline{F D I}, \bar{M}, \hat{\tau})$ in (16). The dashed lines represent the $95 \%$ confidence interval obtained using the bootstrap with 1,000 replications. 


\section{References}

Aghion, P., Howitt, P.(1992), A Model of Growth through Creative Destruction, Econometrica , 60: 423-351

Ahn SC, Good DH, Sickles RC (2000) Estimation of long-run inefficiency levels: A dynamic frontier approach. Econometric Reviews 19:461 - 492.

Ahn SC, Lee YH, Schmidt P (2007) Stochastic frontier models with multiple time-varying individual effects. Journal of Productivity Analysis 27:1-12.

Aigner DJ, Lovell CAK, Schmidt P (1977) Formulation and estimation of stochastic frontier production function models. Journal of Econometrics 6:21-37.

Alcalà F, Ciccone A (2004) Trade and productivity. Quarterly Journal of Economics 119:613-646.

Artis MJ, Galvão ABC and Marcellino M (2007) The Transmission Mechanism in a Changing World. Journal of Applied Econometrics 22:39-61.

Bai J (2009) Panel Data Models with Interactive Fixed Effects. Econometrica $77: 1229-1279$.

Balk BM (2003) The residual: On monitoring and benchmarking firms, industries, and economies with respect to productivity. Journal of Productivity Analysis 20:5-47.

Barro R (2001) Human capital and growth. American Economic Review 91:1217 .

Barro RJ, Sala-i-Martin X (2004) Economic Growth. Second edition, The MIT Press, Mass.

Battese GE, Coelli TJ (1988) Prediction of firm-level technical efficiencies with a generalised frontier production function and panel data. Journal of Econometrics 38:387-399.

Battese GE, Coelli TJ (1995) A model for technical inefficiency effects in a stochastic frontier production function for panel data. Empirical Economics 20:325-332.

Bernard AB, Jones CI (1996) Productivity across industries and countries: Time series theory and evidence. Review of Economics and Statistics 78:135-146.

Borensztein E, De Gregorio J, Lee LW (1998) How does foreign direct investment affect economic growth? Journal of International Economics 45:115-135.

Busse M, Groizard JL (2006) Foreign direct investment, regulations and growth. World Bank Policy, Research Working Paper, no. WPS3882

Cameron G, Proudman J, Redding S, (2005) Technological convergence, R\&D, trade and productivity growth. European Economic Review 49:775-807.

Canova F, Dellas H (1993) Trade interdependence and the international business cycle. Journal of International Economics, 34:23-47.

Caves DW, Christensen LR, Diewert EW (1982) The economic theory of index numbers and the measurement of input, output, and productivity. Econometrica 
50:1393-1414.

Chudik A, Pesaran MH, Tosetti E ( 2011) Weak and strong cross-section dependence and estimation of large panels. The Econometrics Journal 14:C45C90.

Coe DT, Helpman E (1995) International R\&D spillovers. European Economic Review 39:859-887.

Cohen WM, Levinthal DM (1989) Innovation and learning: The two faces of R\&D. Economic Journal 99:569-96.

Cornwell C, Schmidt P, Sickles R (1990) Production frontiers with cross-sectional and time-series variation in efficiency levels. Journal of Econometrics 46:185-200.

Croux C, Forni M, Reichlin L (2001) A measure of comovement for economic variables: Theory and empirics. Review of Economics and Statistics 83:232-241.

Daniels JP, Nourzad F, VanHoose DD (2005) Openness, central bank independence, and the sacrifice ratio. Journal of Money, Credit and Banking 37:371-379.

ECB (2009) Assessing Global Trends in Protectionism. European Central Bank Monthly Bulletin.

Edwards S (1998) Openness, productivity and growth: What we really know?. The Economic Journal 108:383-398.

Eichengreen B (2004) Productivity growth, the new economy, and catching up. Review of International Economics 12:243-245.

Frankel J, Romer D (1999) Does trade cause growth? American Economic Review 89:373-399.

Garrett TA (2003) Aggregated versus disaggregated data in regression analysis: implications for inference. Economics Letters 81:61-65.

Greenwood-Nimmo M, Nguyen VH, Shin Y (2011) Probabilistic forecasting of output growth, inflation and the balance of trade in a GVAR. Forthcoming in Journal of Applied Econometric.

Grossman, G.M., Helpman, E. (1991) Innovation and Growth in a Global Economy, MIT Press, Cambridge

Grossman, G.M., Helpman, E. (1996) Technology and Trade, in G. Grossman and K. Rogoff (eds), Handbook of International Economics, vol. III, Amsterdam, North-Holland.

Hall RE, Jones CI (1999) Why do some Countries produce so much more output per worker than others? Quarterly Journal of Economics 114:83-116.

Hodrick R, Prescott EC (1996) Postwar U.S. business cycles: An empirical investigation. Journal of Money, Credit, and Banking 29:1-16.

Iyer KG, Rambaldi AN, Tang KK (2008), Efficiency externalities of trade and alternative forms of foreign investment in OECD countries. Journal of Applied Econometrics 23:749-766.

Jovanovic B, Rob R (1989) The growth and diffusion of knowledge. Review of Economic Studies 56:569-582. 
Kapetanios G, Pesaran MH (2007) Alternative approaches to estimation and inference in large multifactor panels: Small sample results with an application to modelling of asset returns. in G. Phillips and E. Tzavalis (eds), The Refinement of Econometric Estimation and Test Procedures: Finite Sample and Asymptotic Analysis, Cambridge, Cambridge University Press.

Kneip A, Sickles R, Song W (2011) A new panel data treatment for heterogeneity in time trends. Forthcoming in Econometric Theory.

Kose MA, Prasad EE and Terrones ME (2003) How does globalization affect the synchronization of business cycles? American Economic Review 93:57-62.

Koopmans L (1974) The Spectral Analysis of Time Series. Academic Press, New York, San Francisco, London.

Krugman P (1991) Increasing returns and economic geography. Journal of Political Economy 99:483-99.

Kumbhakar S (1990) Production frontiers, panel data, and time-varying technical inefficiency. Journal of Econometrics 46:201.212.

Kumbhakar SC, Ghosh S, McGuckin JT (1991) A generalized production frontier approach for estimating determinants of inefficiency in U.S. dairy farms. Journal of Business and Economic Statistics 9:279-86.

Kumbhakar S, Lovell CAK (2000) Stochastic Frontier Analysis. Cambridge University Press.

Lee HL (1991) Panel Data Models with Multiplicative Individual and Time Effects: Applications to Compensation and Frontier Production Functions, Ph.D. Dissertation.

Lee HL and Schmidt P (1993) A Production Frontier Model with Flexible Temporal Variation in Technical Inefficiency in the Measurement of Productive Efficiency: Techniques and Applications, in Fried H, Lovell CAK and Schmidt S, Oxford University Press.

Malley JR, Muscatelli VA, Woitek U (2005) Real business cycles or sticky prices? The impact of technology shocks on US manufacturing. European Economic Review 107:745-760.

Mankiw G, Romer D, Weil D (1992) A contribution to the empirics of economic growth. Quarterly Journal of Economics 107:407-437.

Mastromarco C, Woitek U (2007) Regional business cycles in Italy. Computational Statistics \& Data Analysis 52:907-918.

Mastromarco C, Woitek U (2009) A stochastic frontier model with time-varying vector autoregressive inefficiency. Working Paper University of Salento (Lecce).

Meeusen W, van den Broeck J (1977) Efficiency estimation from Cobb-Douglas production functions with composed error. International Economic Review 18:435444.

Nelson R, Phelps E (1996) Investment in humans, technological diffusion, and 
economic growth. American Economic Review: Papers and Proceedings 51:69-75.

OECD (2006) Compendium of Productivity Indicators.

Park B, Sickles R, and Simar L (2007) Semiparametric efficient estimation in dynamic panel data models. Journal of Econometrics 136:281-301.

Pesaran MH (2004) General diagnostic tests for cross section dependence in panels. Cambridge Working Paper in Economics 0435.

Pesaran MH (2006) Estimation and inference in large heterogeneous panels with a multifactor error structure. Econometrica 74:967-1012.

Pesaran MH, Shin Y (1998) Generalized impulse response analysis in linear multivariate models. Economics Letters 58:17-29.

Priestley M, (1981) Spectral Analysis and Time Series. Academic Press, London.

Romer PM (1990) Endogenous technological change. Journal of Political Economy 98:71-102.

Schmidt P, Sickles RC (1984), Production Frontiers and Panel Data, Journal of Business and Economic Statistics 2: 367-74.

Segerstrom P (1991) Innovation, Imitation and Economic Growth. Journal of Political Economy 99:807-827.

Serlenga L, Shin Y (2007a) Gravity models of intra-EU trade: Application of the CCEP-HT estimation in heterogeneous panels with unobserved common time-specific factors. Journal of Applied Econometrics 22:361-381.

Serlenga L, Shin Y (2007b) Monte Carlo studies on finite sample performance of the CCEP-HT estimator in panels with heterogeneous unobserved common factor. The online supplement to the Journal of Applied Econometrics.

Taylor C (2008) Foreign direct investment and the Euro: the first five years. Cambridge Journal Economics 32:1-28.

Tsionas EG (2006) Inference in dynamic stochastic frontier Models. Journal of Applied Econometrics 21:669-676.

Tybout JR (1992) Linking trade and productivity: New research directions. World Bank Economic Review 6:189.211.

Van Ark BO, Mahony M, Ypma G (2007) The EU KLEMS productivity report: Technical report, European Commission. Policy Support and Anticipating Scientific and Technological Needs.

Wacziarg R, Welch KH (2008) Trade liberalization and growth: New evidence. World Bank Economic Review 22: 187-231.

Wang H, Schmidt P (2002) One-Step and Two-Step Estimation of the Effects of Exogenous Variables on Technical Efficiency Levels, Journal of Productivity Analysis, 18, 129-144. 